\title{
Cierre de Escuelas en Contextos Vulnerables desde la Perspectiva de los Orientadores: Impacto en Zonas Rurales
}

\author{
School Closings in Vulnerable Contexts from the Counsellors' \\ Point of View: Impact in Rural Areas
}

\author{
Carlos Monge ${ }^{1 *}$ \\ Patricia Gómez Hernández ${ }^{1}$ \\ Teresa Jiménez Arenas ${ }^{2}$
}

${ }^{1}$ Universidad Complutense de Madrid, España

${ }^{2}$ Consejería de Educación, Cultura y Deportes de Castilla-La Mancha, España

\begin{abstract}
El sistema educativo, al igual que otros ámbitos, se está viendo alterado como consecuencia del Covid-19. Una de las medidas más destacadas es el cese de la actividad presencial en las aulas y su sustitución por la educación a distancia, derivando en un gran impacto en sectores vulnerables, entre los que se encuentran los centros rurales y la atención a la diversidad en la España vaciada. El principal objetivo de esta investigación fue describir y comprender cómo los orientadores escolares de zonas rurales percibían el impacto que tiene la situación sanitaria del Covid-19 en los procesos de enseñanza-aprendizaje. Para ello, se desarrollaron entrevistas como técnica de recogida de información para comprender y dar voz a los orientadores de los Centros Rurales Agrupados de la Provincia de Guadalajara, con el fin de profundizar en los cambios producidos en estas escuelas rurales. Los principales resultados apuntaron que, aunque la adaptación a la virtualidad se ha realizado, fueron muchas las barreras que separaban al sector rural de las grandes áreas urbanas, siendo una vez más una zona castigada y limitada en cuanto a recursos disponibles. En este sentido, se han de dar propuestas concretas para este sector que garanticen una educación de calidad para todos.
\end{abstract}

Descriptores: Atención a la diversidad; Escuela rural; Covid-19; Orientación educativa.

\begin{abstract}
Just like other fields, educational system is being modified as a result of Covid-19. One of the most outstanding measures is the fact that classroom activity has stopped and it has been replaced by distance learning, which may cause a great impact on vulnerable areas such as rural schools and attention to diversity in the so-called emptied Spain. This research aims at describing and understanding how school counsellors in rural areas perceive the impact on teaching-learning processes because of the Covid-19 health situation. To this end, interviews were performed as a gathering information technique for both understanding and giving a voice to counsellors belonging to Centros Rurales Agrupados de la Provincia de Guadalajara (Grouped Rural Centres in province of Guadalajara), in order to further study the changes in these rural schools. The main results pointed out that, despite a certain virtualization has been done, many barriers still isolated the rural areas from the large urban areas, once again being rural areas the most badly affected and resourceconstrained. In this regard, specific proposals must be made for this area for ensuring quality education for everyone.
\end{abstract}

Keywords: Attention to diversity; Rural school; Covid-19; School counselling.

*Contacto: camonge@ucm.es

ISSN: 2254-3139

www.rinace.net/riejs/

revistas.uam.es/riejs
Recibido: $\quad 15$ de junio 2020

$1^{\text {a }}$ Evaluación: 13 de octubre 2020

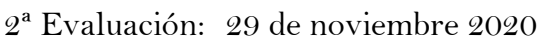

Aceptado: 16 de diciembre 2020 


\section{Introducción}

La situación generada por el Covid-19 tiene un gran impacto en distintos ámbitos de la vida de las personas, de las organizaciones y de las sociedades, ya sea en el ámbito sanitario, laboral, económico, relacional o, entre otros muchos, el educativo. Concretamente en el contexto español, a partir de la entrada en vigor del Real Decreto 463/2020, de 14 de marzo, por el que se declara el estado de alarma para la gestión de la situación de crisis sanitaria ocasionada por el Covid-19, se suspende la actividad educativa presencial en todos los centros y etapas y se sustituye, en la medida de lo posible, por actividades educativas a distancia y online. Esto genera un cambio importante en la enseñanza y en el aprendizaje, pero ese impacto se acrecienta cuando se trata de colectivos vulnerables. Por desgracia, son numerosos los niños y adolescentes en situación de vulnerabilidad que se ven influidos por esta situación, pudiéndose considerar de especial interés el estudio de ese impacto en las zonas rurales, vaciadas, desconectadas de las grandes redes de comunicación y olvidadas económica y políticamente. De igual forma, puede resultar de interés el impacto que tiene esta medida en la atención a la diversidad del alumnado de estas zonas, siendo la figura del orientador quien desempeña un papel fundamental en el asesoramiento, prevención, evaluación e intervención al respecto. Como señala Echeita (2020), es importante detectar y analizar situaciones de vulnerabilidad para intentar aprovechar la pandemia del Covid-19 como una oportunidad de cara a hacer más justos e inclusivos los sistemas educativos.

Según datos de las Naciones Unidas (2020), más de 1.6 billones de estudiantes se ven afectados por el cierre de las escuelas a nivel mundial a causa de esta pandemia, aunque no afecta a todos por igual, siendo más duro en las regiones y colectivos en situación de vulnerabilidad. Bajo esta situación sanitaria, las brechas educativas y la inequidad se ven agravadas (Murillo y Duk, 2020).

Por estas razones, el principal objetivo de esta investigación es describir y comprender cómo los orientadores escolares de zonas rurales perciben el impacto que tiene la situación sanitaria del Covid-19 en los procesos de enseñanza-aprendizaje.

\section{Retos y oportunidades de las escuelas rurales}

Cuando se habla de justicia curricular no se puede pasar por alto que, en el marco de las revoluciones demográficas y estructurales, el sistema educativo no está ideado para el ámbito rural a pesar de las múltiples posibilidades que la educación puede brindar para disminuir la brecha entre lo rural y lo urbano (Torres, 2011). Las transformaciones demográficas, junto a decisiones económicas y políticas, llevan al cierre de escuelas rurales que, en mayor o menor medida, se plasma en alumnos y familias en riesgo de subsistencia, abandono escolar, bajo rendimiento académico y pobreza socio-económica (Núñez et al., 2020). De hecho, los niños y niñas escolarizados en centros educativos rurales son de los primeros colectivos en señalar la invisibilización y desconsideración por parte de la mayoría de políticas públicas hacia las escuelas y entornos rurales (Núñez et al., 2016), aunque ese olvido también es un tema recurrente entre el profesorado (Álvarez-Álvarez y Vejo-Sainz, 2017; Sepúlveda y Gallardo, 2011). Si bien es cierto que el interés de las personas que viven en grandes áreas urbanas se ve incrementado hacia los valores ecológicos, productos, alimentos o entornos paisajísticos del ámbito rural, también cabe destacar el olvido percibido en las políticas públicas hacia el sector educativo en estos 
lugares (Bustos, 2009). El cierre de las escuelas rurales viene siendo un tema de debate en el contexto iberoamericano (Juárez, 2019), aunque la producción científica interesada específicamente en esta cuestión es escasa y con poco poder de generalización (Raso, Sola e Hinojo, 2017), siendo prácticamente inexistente en relación con la situación sanitaria actual de Covid-19. En este sentido, ya existen algunas iniciativas para la investigación del efecto de esta pandemia en los centros educativos (Luengo y Manso, 2020), pero no se recogen las voces de todos los colectivos ni aparece la escuela rural como elemento de análisis.

Además del cierre de escuelas por falta de niños y niñas y problemas de rentabilidad económica, la escuela rural se enfrenta a múltiples retos en diferentes áreas. Una de ellas es la deficiente formación del profesorado para trabajar en estos contextos de vulnerabilidad y para acceder a determinados recursos formativos (Piña, 2014). Otro reto es la gran movilidad del profesorado en estos centros educativos, dificultando así el sentimiento de pertenencia y la integración de los símbolos culturales más cercanos en el currículo (Bustos, 2009). A ello puede sumarse, entre otros muchos retos, el mantenimiento deficiente de algunas infraestructuras e instalaciones (Álvarez-Álvarez y Vejo-Sainz, 2017), la dificultad para escapar de ciertas situaciones de acoso entre iguales (Ruíz-Ramírez et al., 2018), la compleja coordinación con otros servicios y administraciones cuyas sedes se encuentran a grandes distancias del medio rural (Torres, 2011), el complicado liderazgo de proyectos colaborativos en aulas unitarias (MirandaCalderón y Rosabal-Vitoria, 2018), el difícil acceso a internet (Álvarez-Álvarez y VejoSainz, 2017) o la falta de agentes socio-económicos y educativos con carácter dinamizador (Sepúlveda y Gallardo, 2011).

Sin embargo, si bien es cierto que este es un contexto bastante generalizado en el entorno rural, hay realidades que se escapan de esta descripción, pues no todos los entornos rurales son iguales ni todas las escuelas rurales poseen las mismas características. La idea de escuela rural "se amplía enormemente, ya que incluye a municipios que, cuantitativa y cualitativamente, son diferentes, aunque sean coincidentes en su actividad principal. En consecuencia, $[\ldots]$ va a ser muy difícil, cuando no imposible, delimitar conceptualmente este tipo de centros educativos" (Corchón, 2005, p. 73).

A pesar de este abandono sufrido en las escuelas rurales de forma generalizada, también cabe señalar algunas posibilidades que brinda el contexto en ese marco educativo. No se puede olvidar que la escuela rural es un claro articulador de la identidad social con un carácter público, en el sentido de abordar necesidades y valores de la ciudadanía, servir a la comunidad en la que se emplaza y cimentar pedagogías inclusivas (Núñez et al., 2020). La escuela rural potencia un capital social al servicio de la comunidad, donde se llega a generar un fuerte sentimiento de lucha, movilización y compromiso ante situaciones de injusticia económica y política por su desmantelamiento (Núñez et al., 2016).

Algunas de las posibilidades que especialmente ofrece la escuela rural son, entre otras, una mayor atención personalizada por parte del docente (Bustos, 2010; Montalvo y Guillén, 2013), la estrecha relación entre la perspectiva ecológica de los textos expositivos y los conocimientos previos de los niños y niñas (Amado y Borzone, 2012), los conocimientos sobre flora y fauna de estos estudiantes y su inclusión en el currículo de Ciencias Naturales (Hernández-Barbosa, 2018), la mayor autonomía de los profesionales de la educación (Corchón, 2005), las posibilidades de participación en la vida de estos centros educativos 
(Raso, Sola e Hinojo, 2019) o la heterogeneidad de sus aulas como elemento enriquecedor de los aprendizajes (Supúlveda y Gallardo, 2011).

Como recogen Hinojo, Raso e Hinojo (2010), frente a la llamada "vieja escuela rural", la "nueva escuela rural" ofrece ciertas posibilidades como la comunicación virtual entre centros educativos, el acceso a materiales remotos de aprendizaje, el establecimiento de comunidades profesionales de aprendizaje en formato virtual o, entre otras alternativas, la desprivatización de las prácticas docentes.

La escuela rural debe ser vista como un espacio lleno de oportunidades que debe afrontar nuevos y viejos retos, considerando esta situación como una ocasión para transformar y mejorar educativamente, especialmente en cuestiones relacionadas con la participación, la equidad y la convivencia (Rayón y Heras, 2012). Precisamente en estas materias, los orientadores escolares desempeñan un papel fundamental.

\section{El papel del orientador escolar en entornos rurales}

En materia de atención a la diversidad, el orientador escolar viene desarrollando un papel imprescindible, siendo especialmente relevante en contextos de vulnerabilidad, como es el caso de las zonas rurales por las razones esgrimidas anteriormente. A pesar de ello, en algunas regiones españolas se produce una clara deficiencia en este aspecto, considerando que los Equipos de Orientación de zona pueden llegar a brindar una escasa atención directa a las escuelas rurales (Bustos, 2010). En ciertas Provincias con bajas tasas de densidad poblacional, las medidas de orientación académica suponen un claro recurso con impacto en la inclusión educativa, pero todavía necesitan seguir mejorando con medios materiales, técnicos, personales y económicos (Montalvo y Guillén, 2013), lo que coincide con otros resultados encontrados más allá del contexto español (Gurianova, 2014; Hines, 2013; Modiba y Sefotho, 2019).

En algunos territorios, como en el que se enmarca esta investigación, la planificación regional de la orientación en áreas rurales implica distintos niveles y estructuras fundamentales que van desde las Consejerías con competencias en educación hasta los Equipos/Departamentos de Orientación y tutores, pasando por instituciones específicas como los Centros de Recursos y Asesoramiento a la Escuela Rural (Ayala, Molina y Prieto, 2012). No obstante, el desmantelamiento de estos últimos y determinadas políticas públicas llevan a que se perciba una deficiente formación para desarrollar un liderazgo basado en la equidad en el medio rural en áreas que los asesores están descuidando y asumiendo directivos y orientadores (Miranda-Calderón y Rosabal-Vitoria, 2018). En esta confusa configuración de roles, las características de los entornos rurales hacen que, habitualmente, algunas funciones de la orientación se solapen o sean asumidas por los propios profesores-tutores (Harkaitz-Zubiri, Intxausti y Odriozola, 2017).

Algunas prácticas que se pueden considerar de éxito para la inclusión educativa en zonas rurales coinciden en manifestar el alto compromiso de los orientadores escolares y sus habilidades comunicativas, sus competencias para asesorar al profesorado ante situaciones imprevistas y, entre otras cuestiones, las estrategias que emplean para procurar una mejor inserción socio-laboral del alumnado más vulnerable (Ritacco, 2011). De igual forma, una experiencia con alumnos con diversidades conductuales y emocionales en zonas rurales recoge algunas claves desarrolladas por los orientadores con un impacto positivo en su inclusión socio-educativa en el marco de las corrientes de la psicología positiva, como son 
el asesoramiento individualizado para desarrollar nuevas habilidades que faciliten sobrevenirse a los problemas (Thornton, 2018).

A pesar de la importancia de la orientación en las escuelas rurales, se trata de una cuestión compleja que requiere afrontar ciertos retos. En este sentido, desde la visión de los protagonistas directos, Modiba y Sefotho (2019) analizan y recogen algunas percepciones en torno a las dificultades alrededor del acceso y uso de recursos tecnológicos, de las comunicaciones y de la formación docente. Precisamente, desde una visión positiva de esta situación, Hines (2013) reivindica el papel que juegan estos y otros retos como fuerza impulsora para la transformación de la orientación educativa en contextos rurales. Ante la deslocalización progresiva de las escuelas rurales, algunas propuestas pasan por el diseño de programas virtuales de asesoramiento desde los profesionales de la orientación educativa, pero las dificultades de acceso a internet y la escasa formación siguen siendo evidentes en estos contextos (Johnson y Daire, 2008).

Por todo ello, resulta necesario seguir investigando y proponiendo respuestas que ayuden a mejorar estas situaciones, especialmente en los colectivos más vulnerables.

\section{Método}

Esta investigación se basó en el uso de la entrevista como técnica para comprender y dar voz a los orientadores de las escuelas rurales de la Provincia de Guadalajara, donde la tasa de densidad poblacional es baja y la despoblación es cada vez mayor. De hecho, las peculiaridades de ciertos territorios de esta Provincia han hecho que fuse de interés para estudios previos sobre inclusión educativa (Montalvo y Guillén, 2013), formación docente (Piña, 2014) y convivencia escolar (Rayón y Heras, 2012).

\section{Participantes}

En este estudio participaron cinco orientadores de Centros Rurales Agrupados de la Provincia de Guadalajara, los cuales ofrecían un servicio a alumnos de Educación Infantil y Primaria. Si bien es cierto que el número de participantes fue reducido, cabe destacar que, como ha sucedido en otros estudios previos (Modiba y Sefotho, 2019), los escasos recursos personales de la orientación educativa en las zonas rurales obligaron a considerar un número pequeño de entrevistados, teniendo en cuenta también que lo importante aquí no fue generalizar los resultados a la población a partir de una muestra representativa, sino poder analizar y comprender las subjetividades de los participantes.

\section{Técnicas de recogida de información}

Dadas las posibilidades que las entrevistas han brindado para descubrir y comprender las ideas y prácticas de colectivos educativos inmersos en zonas rurales (Álvarez-Álvarez y Vejo-Sainz, 2017) y para visibilizar situaciones desfavorables de la orientación en entornos rurales (Modiba y Sefotho, 2019), en esta investigación se optó por esta técnica de recogida de datos. Concretamente, se realizaron cuatro entrevistas a cada participante a lo largo de 20 días:

- La primera versó sobre los cambios organizativos y curriculares adoptados ante el cese de la actividad presencial en los centros educativos.

- La segunda de las entrevistas lo hizo sobre el impacto de dichos cambios y el propio cierre de los centros educativos. 
- La tercera entrevista se centró en la participación y colaboración de los diversos colectivos de la comunidad educativa durante esta situación sanitaria.

- Y la cuarta entrevista abordó las principales facilidades y dificultades para adaptarse a esta nueva situación en los Centros Rurales Agrupados.

\section{Procedimiento}

A partir del listado de Centros Rurales Agrupados de la Provincia de Guadalajara recogidos en la Orden 22/2020, de 12 de febrero, de la Consejería de Educación, Cultura y Deportes de Castilla-La Mancha, se procedió a contactar telemáticamente con los equipos directivos de cada uno de ellos solicitando el respaldo institucional y la colaboración del centro para la investigación. Dichos equipos directivos enviaron la información correspondiente a los orientadores escolares de tales centros educativos. De los 12 Centros Rurales Agrupados guadalajareños, cinco de los orientadores finalmente accedieron a ser entrevistados.

Dada la situación de emergencia sanitaria generada por el Covid-19, las entrevistas tuvieron que realizarse de forma online, las cuales fueron analizadas siguiendo las características de la Teoría Fundamentada (Glaser y Strauss, 2017): muestreo teórico, saturación teórica y categorización (abierta, axial y selectiva). Para el análisis de la información se recurrió al programa Atlas.ti, considerando que en las citas textuales recogidas en este artículo se añaden dos números proporcionados por este programa, siendo el primero de ellos el referente al número de entrevista y el segundo se correspondió con el número de la cita dentro de ese documento.

Antes de iniciar las entrevistas, todos los participantes fueron informados por escrito de los objetivos de la investigación, de la voluntariedad de participación y del tratamiento ético de los datos por parte del equipo investigador. En este sentido, a lo largo de este trabajo se ha evitado hacer alusión directa a las características personales y profesionales de los participantes y a los centros educativos, pues, dado el tamaño muestral y la idiosincrasia de las escuelas rurales de este territorio, esto hubiese permitido romper la confidencialidad de los informantes.

\section{Resultados}

El análisis deductivo arrojó un sistema categorial de análisis con las siguientes grandes familias de categorías emergentes (Figura 1): cambios producidos en las escuelas rurales tras su cierre, participación escolar, impacto de las actividades educativas y valoraciones asociadas a estos fenómenos. El análisis cualitativo de las respuestas de los participantes mostró diferentes informaciones relevantes sobre el impacto del Covid-19 en los Centros Rurales Agrupados de la Provincia de Guadalajara. 


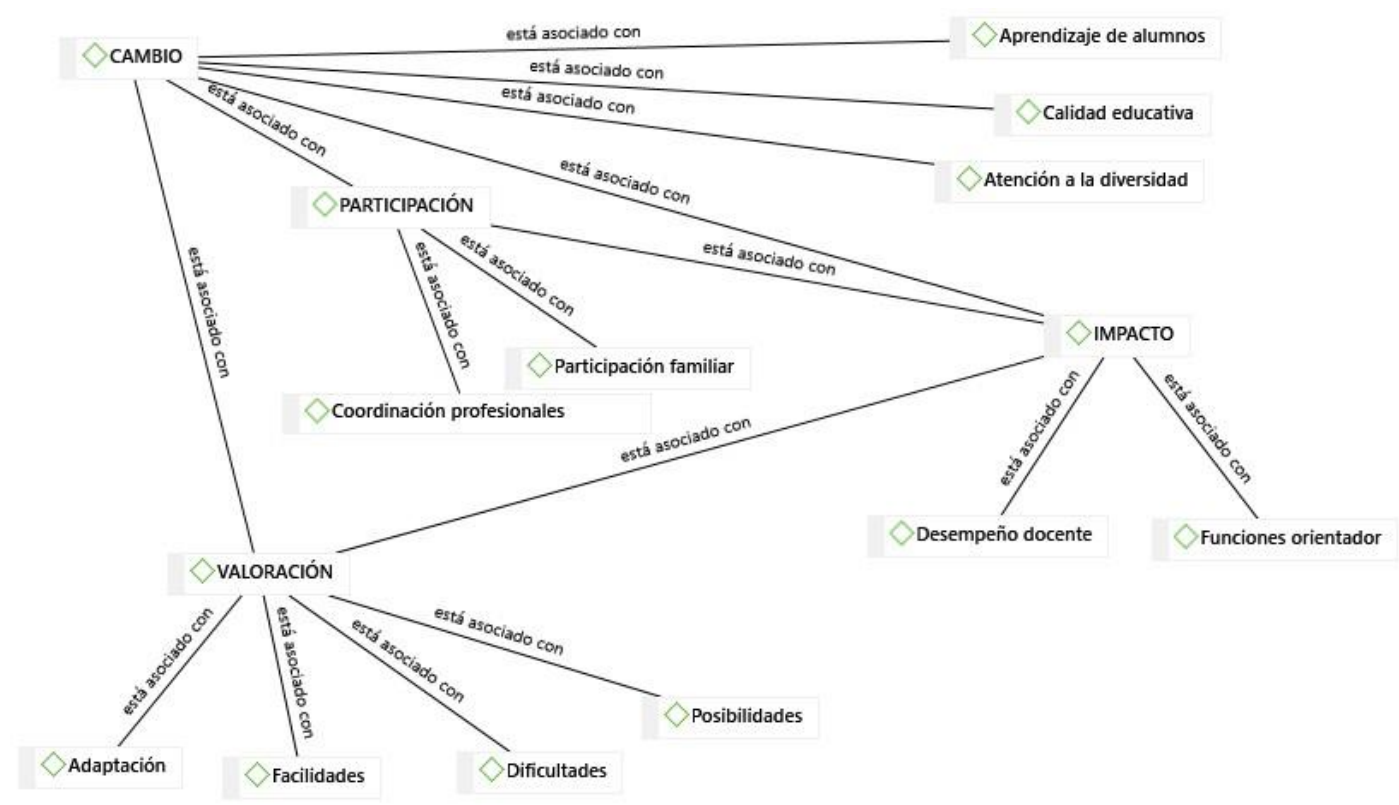

Figura 1. Sistema emergente de análisis de las entrevistas Fuente: elaboración propia

\subsection{Cambios producidos tras el cierre de las escuelas rurales}

Los cambios producidos en estos centros educativos tras la finalización de la enseñanza presencial, como consecuencia de la situación sanitaria generada por el Covid-19, se relacionaron especialmente con los aprendizajes del alumnado, con la calidad educativa y con la atención a la diversidad.

En cuanto a la dimensión de los aprendizajes de los alumnos, se pudo destacar que se han adoptado medidas durante el confinamiento, principalmente mediante el uso de recursos telemáticos, tanto para facilitar los aprendizajes como para promover la coordinación entre el equipo docente, las familias y el alumnado:

Las medidas que ha adoptado el centro durante el confinamiento se basan en sistemas telemáticos para continuar con las clases y facilitar la comunicación entre todos los implicados en el proceso de enseñanza-aprendizaje, especialmente profesorado, alumnado y familias. (Entrevista, orientador 1, 1:1)

Respecto a la calidad educativa, la mayoría de las repuestas de los participantes argumentaba que la calidad de los aprendizajes era peor en la mayoría de las ocasiones, lo que se atribuyó a que la enseñanza virtual, tal como estaba planteada, acentuaba las desigualdades sociales y económicas del alumnado. Analizando las respuestas se pudo destacar la existencia de una brecha digital y social, donde había familias que no sabían cómo afrontar el aprendizaje de sus hijos.

Sin emoción no hay aprendizaje. El profesorado cuando enseña, además de transmitir conocimientos, transmite valores y emociones. Sin estas cosas no se produce aprendizaje. También se han acentuado las desigualdades en la mayoría de los casos debidas a los contextos sociales y familiares existentes. (Entrevista, orientador 2, $5: 2)$

En relación con la dimensión sobre la atención a la diversidad, se pudo destacar que todos los participantes afirmaron que los alumnos que precisan medidas educativas individualizadas y extraordinarias han sido los más afectados ante esta situación, ya que 
ha sido muy difícil darles una respuesta individualizada y adecuada a sus necesidades. Pese a ello, los Equipos de Orientación han hecho un esfuerzo considerable para atender de la mejor forma posible las necesidades de estos alumnos.

Creo que en este alumnado ha influido más que en otro la respuesta familiar. Algunas sí han estado en contacto con el equipo de orientación, quien se ha volcado con ellos y ellas por tratarse de un colectivo más vulnerable. Pero las familias más reticentes al contacto y trabajo en casa han limitado esta función [...]. En ocasiones es difícil, pero se está haciendo todo lo posible. Se están adaptando los contenidos, actividades... a la situación particular de cada alumno. (Entrevista, orientador 5, 20:6)

\subsection{Participación escolar durante el confinamiento}

A pesar de las distintas formas que tomó la participación en las escuelas rurales de la Provincia de Guadalajara y de los múltiples colectivos participantes, esta categoría de análisis se relacionó especialmente con la contribución de las familias y con la coordinación entre los principales profesionales educativos.

En líneas generales, de la participación de las familias destacó que la mayoría de las respuestas de los orientadores iban encaminadas a señalar que muchas de las familias se han implicado en el proceso de enseñanza-aprendizaje de sus hijos e hijas en la medida de sus posibilidades. También coincidieron en señalar que, debido a la brecha digital, con algunas familias era más difícil contactar, pero se han utilizado otros medios tradicionales (llamadas telefónicas) y novedosos, como los sistemas de mensajería instantánea (WhatsApp).

Sí, las familias se han implicado para que sus hijos pudiesen seguir el proceso de aprendizaje durante esta situación. Sin duda alguna, las familias han sido una pieza importantísima, pues en ocasiones el alumnado no posee autonomía en la realización de las tareas, y estas son las encargadas de ayudarles con explicaciones, utilización de tecnologías y redes sociales. (Entrevista, orientador 5, 20:3)

Paralelamente, todos los orientadores entrevistados afirmaron que la coordinación entre los distintos profesionales de la educación ha sido satisfactoria a pesar de la situación sanitaria, aunque lo que había cambiado fue el modo en el que se realizaba esa coordinación. En este caso, se ha pasado de reuniones presenciales de coordinación a sesiones y comunicaciones telemáticas.

Por supuesto que se ha modificado el contacto y la coordinación entre la orientación y los docentes durante este periodo especial, pero [...] tenemos herramientas que nos han facilitado el contacto y la coordinación, como son Microsoft Teams o Google Meet.

(Entrevista, orientador 1, 2:7)

\subsection{Impacto de las tareas educativas en modalidades telemáticas}

Si bien es cierto que el Covid-19 ha modificado múltiples formas de actuar y desarrollar las funciones de los profesionales de la educación, durante las entrevistas se relacionaron estrechamente con el desempeño docente y con el papel de los orientadores escolares.

En el caso del desempeño docente, los participantes en esta investigación tuvieron visiones diferentes sobre si el profesorado había sabido adaptarse a los nuevos cambios en el modelo de enseñanza. Varios participantes comentaron que el profesorado se había adaptado a la nueva realidad, considerando que algunos aprendieron a utilizar nuevas herramientas y materiales tecnológicos, y otros lo hicieron utilizando herramientas innovadoras que ya utilizaban en el modo presencial: "sí, han sabido adaptarse a los cambios en el modelo de enseñanza exigido por el Covid-19, pero han supuesto mucho más esfuerzo y horas de dedicación para ellos" (Entrevista, orientador 3, 12:4). Sin embargo, otros orientadores 
entrevistados destacaron que el profesorado no se había podido adaptar a la nueva realidad porque no tenía un sistema claro y viable de planificación curricular: "creo que no han sabido adaptarse. No hay una coordinación que permita no sobrecargar a los alumnos de tareas. Muchos pretenden llevar el ritmo normal que, en la enseñanza presencial, y eso es imposible" (Entrevista, orientador 5, 22:4). Pudo destacarse que los resultados de esta dimensión dependieron sustancialmente de las aptitudes del profesorado, de la formación docente recibida y de la organización y coordinación por parte del equipo docente y del equipo directivo.

De igual forma, las funciones y actividades de los orientadores de las escuelas rurales también fueron cambiando según se iban desarrollando los acontecimientos sanitarios y académicos. Especialmente, algunas funciones que estaban vinculadas a la orientación académica y profesional y al asesoramiento psicopedagógico a las familias y a los profesores se vieron afectas, teniendo que ser adaptadas a la modalidad telemática. En cambio, el desarrollo de las funciones relacionadas con las evaluaciones psicopedagógicas y la detección de barreras y potencialidades del aprendizaje han sido, según los participantes en esta investigación, imposibles de realizar adecuadamente.

\begin{abstract}
La respuesta del equipo se ha centrado en dar herramientas y recursos para que el seguimiento del proceso de enseñanza-aprendizaje y el cuidado emocional siguiera adelante, viéndose afectado lo menos posible. Pero se han tenido que hacer ajustes, dejando de lado las evaluaciones del alumnado derivado por el equipo docente con barreras para el aprendizaje. (Entrevista, orientador 4, 16:7)

Como el resto del personal educativo, nos estamos adaptando a esta modalidad a distancia, facilitando recursos y procurando la coordinación entre todos los implicados en educación, pero sí que es cierto que parte de nuestras funciones, como puede ser la observación, pasar pruebas estandarizadas o estudio de trabajos, no son posibles ahora. (Entrevista, orientador 1, 3:7)
\end{abstract}

\title{
5.4. Valoraciones asociadas a los cambios producidos tras el cese de la actividad presencial
}

Las principales valoraciones ofrecidas por los orientadores de las escuelas rurales guadalajareñas se centraron principalmente sobre las posibilidades de las adaptaciones realizadas, las dificultades encontradas, las facilidades halladas y las potencialidades, poniendo especial atención sobre cómo las características de los entornos y escuelas rurales influyen sobre dichas ideas.

En primer lugar, la mayoría de los participantes confirmaron que la conectividad de los entornos rurales fue menor que la encontrada en las zonas urbanas, lo que ha producido mayores dificultades en los actores y agentes educativos para acceder a recursos para el aprendizaje:

Las redes de conexión no llegan del mismo modo. No hay una calidad en ellas y eso afecta al trabajo diario de docentes, familias y alumnado. (Entrevista, orientador 2, 8:9)

En cambio, otros participantes destacaron que las escuelas rurales podrían tener las mismas posibilidades que el resto de centros educativos y que, más bien, sería un problema de brecha digital y un adecuado conocimiento de las tecnologías por parte de las familias:

Creo que, frente al acceso a internet, influye más del conocimiento, o desconocimiento, de las herramientas tecnológicas por parte de las familias. (Entrevista, orientador 4: 17:11) 
Las dificultades con las que se encontraron los orientadores de las escuelas rurales dependieron de la situación y de las necesidades de cada centro educativo. A pesar de eso, también han existido ciertas dificultades compartidas:

- El aprendizaje rápido por parte del profesorado en torno a la competencia digital.

- Dificultades para conseguir que el aprendizaje y la información llegasen a todo el alumnado.

- Dificultades para coordinarse con las familias y con el equipo docente por medio telemático.

- Falta de recursos y medios tecnológicos por parte de las familias.

En relación con las facilidades que ha tenido la escuela rural en esta situación, las respuestas fuero bastante dispares. Entre ellas se pudieron encontrar aspectos como:

- La formación intensiva sobre nuevas tecnologías.

- La implicación y el esfuerzo del equipo docente.

- El intercambio constante de nuevos materiales y recursos tecnológicos.

En cuanto a las posibilidades que tuvieron las escuelas rurales para continuar con el proceso de formación a distancia, las respuestas de los participantes se dividieron en dos grandes grupos. Por un lado, se situaron aquellos orientadores que comentaban la existencia de mayores dificultades para seguir una formación a distancia en las escuelas rurales debido a la conectividad y al bajo nivel educativo de algunas familias. Y por otro lado, se encontraban aquellos participantes entrevistados con respuestas coincidentes en que las limitaciones y fortalezas de la educación a distancia eran las mismas que en las zonas urbanas.

Creo que el modelo de formación que se ha adoptado durante este periodo, en ciertas etapas educativas (Educación Infantil y Educación Primaria), en general, tiene muchas dificultades, pero en el entorno rural estas dificultades son aún mayores. Es un modelo que dificulta el acceso a nuevos aprendizajes y sacrifica gran parte de los contenidos del currículo de estas etapas. (Entrevista, orientador 1, 4:12)

En nuestro caso, los problemas técnicos se solucionaron y, hoy en día, en este Centro Rural Agrupado pueden tener las mismas posibilidades que en una ciudad. Incluso, tras la vuelta a las clases presenciales, será una ventaja por las ratios pequeñas y los espacios más grandes. (Entrevista, orientador 4, 19:11)

\section{Discusión y conclusiones}

La situación sanitaria derivada del Covid-19 produce innumerables consecuencias en la vida de las personas, de las organizaciones y de las sociedades. Concretamente, en el ámbito educativo provoca, entre otras cuestiones, el cierre de las escuelas a nivel mundial y un impacto incalculable a largo plazo de sus consecuencias (Naciones Unidades, 2020), pero esta situación se agrava más cuando se trata de colectivos vulnerables y en riesgo de exclusión (Echeita, 2020; Murillo y Duk, 2020). En este caso, sin pretender ser excluyentes, se puede considerar a la escuela rural como una institución vulnerable ante determinadas problemáticas (Núñez et al., 2020) y ante el olvido que sufre por parte de determinadas administraciones (Torres, 2011). Sin duda, cuando se trata de contextos vulnerables, el papel que desempeñan los orientadores de las escuelas rurales resulta 
fundamental para la inclusión educativa (Bustos, 2009; Gurianova, 2014; Hines, 2013; Modiba y Sefotho, 2019; Montalvo y Gullén, 2013).

Coincidiendo con otras investigaciones (Juárez, 2019; Raso, Sola e Hinojo, 2017), los resultados aquí hallados vienen a poner de manifiesto la pérdida de calidad en los procesos de enseñanza-aprendizaje que se produce ante el cierre de las escuelas rurales en la Provincia de Guadalajara. Por ello, a la vuelta de la normalidad sanitaria se debe ser vigilante para evitar la clausura de estos centros y para que la modalidad telemática no sea una vía de escape de cara a proponer alternativas que lleven a la desaparición de la escuela rural en este territorio, como se da por algunas situaciones migratorias (Bustos, 2010; Montalvo y Guillén, 2013) y ciertas políticas económicas (Torres, 2011).

A pesar de las dificultades encontradas ante el cierre de las escuelas rurales por causa del Covid-19, los resultados de esta investigación muestran algunos de los esfuerzos que realizan los Equipos de Orientación para atender a la diversidad en la Provincia de Guadalajara. En este caso, cabe considerar que el compromiso de los orientadores de las escuelas rurales guadalajareñas es un elemento básico de cara a promover la inclusión educativa (Ritacco, 2011).

Como se recoge en los resultados hallados, la participación de las familias es un elemento fundamental para el aprendizaje del alumnado en esta región, tanto en escenarios de pandemia como en contextos de normalidad o relativa normalidad. Y es que las escuelas rurales brindan ciertas posibilidades de participación que las escuelas urbanas no hacen (Raso, Sola e Hinojo, 2017), pero no puede olvidarse que las situaciones de pobreza social y económica de las familias influyen sobre la calidad del apoyo dado a sus hijos y que la brecha educativa y las desigualdades pueden verse acrecentadas ante el cierre de las escuelas rurales (Murillo y Duk, 2020; Núñez et al., 2020). De igual forma, los resultados subrayan la importancia que cobra la coordinación entre los distintos profesionales de la educación, siendo capaces de sobreponerse al cese de la actividad presencial en la Provincia, aunque, coincidiendo con otros datos (Modiba y Sefotho, 2019), los sistemas de comunicación basados en plataformas virtuales suponen un verdadero reto en la escuela rural.

Debe tenerse en cuenta que los orientadores participantes en esta investigación destacan la participación de las familias y del resto de compañeros que trabajan codo con codo en las escuelas rurales, pero omiten la coordinación con otros agentes educativos. En línea con lo apuntado por Torres (2011), esto puede deberse a la distancia física que separa las escuelas rurales de las Administraciones educativas situadas en las ciudades. O, según lo propuesto por Sepúlveda y Gallardo (2011), también puede deberse a la falta de agentes socio-económicos y educativos con carácter dinamizador en estos entornos desfavorecidos.

Por otro lado, la situación generada por el Covid-19 conlleva drásticos cambios en las escuelas rurales, incluyendo las tareas desarrolladas tanto por el profesorado como por los orientadores. En cualquier caso, los resultados apuntan que el adecuado desempeño de esas tareas depende en gran medida de la formación inicial y continua de estos profesionales que trabajan a lo largo de la Provincia. A pesar de esa importancia atribuida a la formación, otros estudios coinciden en destacar el débil sistema de formación para los profesionales de las escuelas rurales (Johnson y Daire, 2008; Miranda-Calderón y RosabalVitoria, 2018; Modiba y Sefotho, 2019; Piña, 2014). Por estas razones, resulta imprescindible exigir políticas públicas encaminadas a fortalecer ese sistema formativo en 
estos entornos vulnerables (Ayala, Molina y Prieto, 2012) y, por consiguiente, cabe estar atento ante las políticas educativas que procuran su desmantelamiento.

Según los orientadores entrevistados, algunas tareas de la orientación sí pueden ser trasladadas a entornos telemáticos, pero otras necesariamente han de hacerse de forma presencial. Precisamente, esto coincide con lo apuntado por Johnson y Daire (2008) años atrás y que, actualmente, sigue siendo un reto que asumir en la escuela rural de Guadalajara.

Otro reto apuntado previamente (Álvarez-Álvarez y Vejo-Sainz, 2017; Johnson y Daire, 2008) hace referencia a la conectividad y al acceso a internet, lo que, según algunos orientadores participantes en esta investigación, dificulta considerablemente la enseñanza online. Sin embargo, en línea con Modiba y Sefotho (2019), otros participantes argumentan que la dificultad no está tanto en la accesibilidad, sino que lo está en las competencias tecnológicas de algunas familias y docentes.

No obstante, todas estas conclusiones han de ser tomadas con cautela considerando las debilidades metodológicas de esta investigación. Dado el reducido número de participantes y las características propias de la Provincia de Guadalajara, cabe tener precaución a la hora de transferir los resultados a otros contextos. Por ello, futuras líneas de investigación pueden ir encaminadas a estudiar el cierre de las escuelas rurales en otros territorios con baja densidad poblacional.

En definitiva, lo que se pretende es aportar algunas evidencias que ayuden a visibilizar las problemáticas de la escuela rural y a contribuir a su transformación y mejora.

\section{Referencias}

Álvarez-Álvarez, C. y Vejo-Sainz, R. (2017). ¿Cómo se sitúan las escuelas españolas del medio rural ante la innovación? Un estudio exploratorio mediante entrevistas. Aula Abierta, 45(1), 2532. https://doi.org/10.17811/rifie.45.1.2017.25-32

Amado, B. y Borzone, A. M. (2012). Reading and comprehension in rural schools: The relationship between ecological perspective in expository texts and children's prior knowledge. Culture and Education, 24(1), 17-32. https://doi.org/10.1174/113564012799740821

Ayala, C. L., Molina, V. y Prieto, R. (2012). Los compromisos singulares entre administración y centros para el éxito educativo. Revista de Educación, número extraordinario, 195-219. http://doi.org/10.4438/1988-592X-RE-2012-EXT-2 12

Bustos, A. (2009). La escuela rural española ante un contexto en transformación. Revista de Educación, 350, 449-461.

Bustos, A. (2010). Aproximación a las aulas de escuela rural: Heterogeneidad y aprendizaje en los grupos multigrado. Revista de Educación, 352, 353-378.

Corchón, E. (2005). La escuela en el medio rural: Modelos organizativos. DaVinci Continental.

Echeita, G. (2020). La pandemia del Covid-19. ¿Una oportunidad para pensar en cómo hacer más inclusivos nuestros sistemas educativos? Revista Internacional de Educación para la Justicia Social, 9(1), 7-16. https://doi.org/10.15366/riejs2020.9.1

Glaser, B. G. y Strauss, A. L. (2017). The discovery of grounded theory: Strategies for qualitative research. Routledge. 
Gurianova, M. P. (2014). The development of the institution of school counselors in rural Russia. Russian Education \& Society, 56(9), 82-90. https://doi.org/10.2753/RES1060-9393560905

Harkaitz-Zubiri, J. B., Intxausti, N. y Odriozola, A. (2017). Acción tutorial en la transición del alumnado de las escuelas del medio rural a la educación secundaria. Tendencias Pedagógicas, 29, 189-2 10. https://doi.org/10.15366/tp2017.29

Hernández-Barbosa, R. (2018). Los conocimientos faunísticos en el estudiantado de escuelas rurales: Una guía para su reconocimiento en la clase de ciencias naturales. Educare, 22(2), 119. https://doi.org/10.15359/ree.22-2.1

Hines, P. T. (2013). Transforming the rural school counselor. En S. J. Sears (Ed.), Transforming school counseling: A national perspective (pp. 192-201). Taylor \& Francis.

Hinojo, F. J., Raso, F. y Hinojo, M. A. (2010). Análisis de la organización de la escuela rural en Andalucía: Problemática y propuestas para un desarrollo de calidad. REICE. Revista Iberoamericana sobre Calidad, Eficacia y Cambio en Educación, 8(1), 79-105.

Johnson, N. M. y Daire, A. P. (2008). School counselor implementation of an electronic mentoring (e-mentoring) program in a rural setting. Journal of Technology in Counseling, 5(1), 1-14.

Juárez, D. (2019). Políticas de cierre de escuelas rurales en Iberoamérica. Debates y experiencias. Red Temática de Investigación de Educación Rural.

Luengo, F. y Manso, J. (2020). Informe de investigación Covid-19. Voces de docentes y familias. Proyecto Atlántida.

Miranda-Calderón, L. A. y Rosabal-Vitoria, S. (2018). La gestión directiva en escuelas unidocentes y dirección 1: Un desafío para alcanzar la equidad educativa en contextos rurales de Costa Rica. Educare, 22(3), 1-30. https://doi.org/10.15359/ree.22-3.10

Modiba, M. R. y Sefotho, M. M. (2019). Life orientation teacher training needs in career guidance at rural high schools. South African Journal of Education, 39(2), 1-8. https://doi.org/10.15700/saje.v39ns2a 1538

Montalvo, D. y Guillén, P. (2013). La escuela inclusiva a examen: orientaciones para su mejora. En E. Sebastián, C. R. Mosca y S. E. S. Oliveira (Eds.), La formación del profesorado para la atención a la diversidad en Brasil y España (pp. 81-121). Universidad de Alcalá.

Murillo, F. J. y Duk, C. (2020). El covid-19 y las brechas educativas. Revista Latinoamericana de Educación Inclusiva, 14(1), 11-13. https://doi.org/10.4067/So718-73782020000100011

Naciones Unidas. (2020). Covid-19 and human rights. We are all in this together. Naciones Unidas.

Núñez, C., González, B., Ascorra, P. y Grech, S. (2020). Contar para comprender: Cierre de escuelas rurales municipales en Chile y sus implicaciones para las comunidades. Educação e Sociedade, 41, 1-19. https://doi.org/10.1590/es.215922

Núñez, C., Peña, M., Cubillos, F. y Solorza, S. (2016). Estamos todos juntos: El cierre de la escuela rural desde la perspectiva de los niños. Educação e Pesquisa, 42(4), 953-967.

https://doi.org/10.1590/s1517-9702201612152334

Piña, R. (2014). Desarrollo de la formación permanente del profesorado en la provincia de Guadalajara: un estudio de caso. Enseñanza E Teaching, 32(1), 141-159.

https://doi.org/10.14201/et2014321141159

Raso, F., Sola, T. e Hinojo, F. J. (2017). Satisfacción del profesorado de la escuela rural de la provincia de Granada (España) respecto a la organización escolar. Bordón. Revista de Pedagogía, 69(2), 79-96. https://doi.org/10.13042/Bordon.2017.41372 
Rayón, L. y Heras, A. M. (2012). Una escuela rural en transformación. De una ciudadanía local a una ciudadanía global. Profesorado. Revista de Currículum y Formación del Profesorado, 16(1), 325-343.

Ritacco, M. (2011). Los departamentos de orientación ante la exclusión social: Prácticas inclusivas. Revista Española de Orientación y Psicopedagogía, 22(2), 201-218. https://doi.org/10.5944/reop.vol.22.num.2.2011

Ruíz-Ramírez, R., García-Cué, J. L., Ruíz-Martínez, F. y Ruíz-Martínez, A. (2018). La relación bullying-deserción escolar en bachilleratos rurales. Revista Electrónica de Investigación Educativa, 20(2), 37-45. https://doi.org/10.24320/redie.2018.20.2.1527

Sepúlveda, M. P. y Gallardo, M. (2011). La escuela rural en la sociedad globalizada: Nuevos caminos para una realidad silenciada. Profesorado. Revista de Currículum y Formación del Profesorado, 15(2), 141-153.

Thornton, F. (2018). Counselors and special educators in rural schools working together to create a positive school community. International Electronic Journal of Elementary Education, 10(3), 385-389. http://doi.org/10.26822/iejee.2018336197

Torres, J. (2011). La justicia curricular. El caballo de Troya de la cultura escolar. Morata.

\section{Breve CV de los/as autores/as}

\section{Carlos Monge}

Doctor Internacional en Educación por la Universidad de Alcalá (Premio Extraordinario). Comenzó formándose en la Universidad de Alcalá bajo un contrato predoctoral (FPUUAH) en el Departamento de Ciencias de la Educación dentro del grupo de investigación e innovación "Inclusión y Mejora Educativa: Convivencia y Aprendizaje Cooperativo". Actualmente, es Profesor Ayudante Doctor en el Departamento de Estudios Educativos (área de Didáctica y Organización Escolar) de la Universidad Complutense de Madrid dentro del grupo de investigación "Desarrollo Tecnológico, Exclusión Sociocultural y Educación". Sus publicaciones giran en torno a la innovación educativa, la formación docente y la inclusión educativa. ORCID ID: https://orcid.org/0000-0002-7761-6087. Email: camonge@ucm.es

\section{Patricia Gómez Hernández}

Doctora Internacional en Educación por la Universidad de Alcalá. Comenzó formándose en la Universidad de Alcalá bajo un contrato predoctoral (FPU-UAH) en el Departamento de Ciencias de la Educación. Actualmente, es Profesora Ayudante Doctor en el Departamento de Estudios Educativos (área de Didáctica y Organización Escolar) y miembro de los grupos de investigación "Desarrollo tecnológico, exclusión sociocultural y educación" y "Estudios sobre comunicación y lenguajes para la inclusión y la equidad educativa" de la Universidad Complutense de Madrid. Sus líneas de investigación giran en torno al uso de TIC para la formación docente y la inclusión e innovación educativas. ORCID ID: https://orcid.org/O000-0001-9163-7637. Email: patgom18@ucm.es

\section{Teresa Jiménez Arenas}

Graduada en Pedagogía por la Universidad Complutense de Madrid y Máster en Formación del Profesorado de Educación Secundaria (Orientación Educativa) por la Universidad de Alcalá. Ha ejercido como orientadora en varios institutos y actualmente 
trabaja como orientadora en el CEIP Maestra Teodora de Marchamalo (Guadalajara, CLM). Sus intereses se centran sobre la atención a la diversidad en educación y la orientación académico-laboral. ORCID ID: https://orcid.org/0000-0001-7749-9063. Email: tjimeza@edu.jccm.es 